\title{
Asymptotic modelling of interfaces taking contact conditions into account: Asymptotic expansions and numerical implementation
}

\author{
F. Lebon ${ }^{\mathrm{a}, *}$, F. Zaittouni ${ }^{\mathrm{b}}$ \\ ${ }^{\mathrm{a}}$ Laboratoire de Mécanique et d'Acoustique, Aix-Marseille University-CNRS, 31, Chemin Joseph Aiguier, 13402 Marseille Cedex 20, France ${ }^{\mathrm{b}}$ Laboratoire de \\ Mécanique et Génie Civil, Montpellier 2 University-CNRS, Pl.E. Bataillon, CC048, 34095 Montpellier Cedex 5, France
}

\begin{abstract}
In the present theoretical and numerical study on thin layers, a method is presented for modelling bonding processes and interfaces. When the thickness and the rigidity of the layer tend to zero, the asymptotic problem leads to a limit problem with a mechanical constraint on the surface, to which the layer shrinks. The formulation of the limit problem includes the mechanical and geometrical properties of the layer. The contact and pseudo-friction conditions between the adhesive and the adherents are also taken into account. Theoretical results are obtained using asymptotic expansions and numerical data. The asymptotic laws are implemented in a finite element software. Numerical algorithms are detailed and numerical examples are presented.
\end{abstract}

\section{Introduction}

From an industrial point of view, understanding and modelling interface in structures is crucial. In a large number of assembled structures, interfaces play an essential role in their mechanical behavior. It explains why interface modelling is not recent, in fact it is considered that the first studies are due to Amontons [3] and Coulomb [7]. At least two different approaches are used to study mechanically these interfaces. In the first one (phenomenological), the thickness of the interface is zero and the mechanical properties are obtained from physical considerations. There exists a large class of such models (see for examples $[17,30,15,4,10,2,6,16,5,11,25,14,26,27])$. The second one (deductive), consists in starting with a small thickness (less than $1 \mathrm{~mm}$ in glue-bonding processes), imposing mechanical properties to the interface and studying asymptotically the problem when the thickness tends to zero. We adopt the second approach in this paper and also consider that the interface is soft.

The analysis of soft adhesive bonded joints between two deformable bodies involves problems with several parameters. At least two of these parameters are essential:

- the thickness of the joint, which is small with respect to those of the deformable bodies,

- the stiffness of the joint, which is lower than that of the bodies.

In previous studies (see for example $[1,32,18,22-24,28,20,12,33,19,34]$ ), the bonding of two three-dimensional solids under linear elastic, finite strains and viscoelastic conditions by an adhesive layer has been handled by performing asymptotic analyses, i.e. by assuming the thickness and the stiffness of the joint to tend to zero. With this approach, the layer is replaced

\footnotetext{
* Corresponding author.

E-mail address: lebon@lma.cnrs-mrs.fr (F. Lebon).
} 
by a mechanical constraint. The layer no longer exists from the geometrical point of view, but is replaced by a constraint taking the asymptotic behavior of the parameters into account. From the numerical point of view, this theory is crucial because of the large number of degrees of freedom and the ill-conditioned real problem which it is very hard to solve even in linear cases. However, it is of great importance to obtain conditions on the parameters whenever it is possible to replace the real problem by the limit one.

When the adhesive is linearly elastostatic, the local form of the limit problem is subject to a transmission condition linking the stress vector to the displacement jump occurring at the interface. The structure of the contact law is similar in this case to that of the original constitutive equation, but the strain tensor is replaced by the tensorial product of the displacement jump multiplied by the unit normal vector.

In this paper, this theory is extended to static frictional contact conditions (Signorini and pseudo-Coulomb laws) between the layer and the deformable bodies. The new conditions of transmission are regularized frictional contact (compliance) laws. The multivalued nature of the problem can thus disappear and some classical laws are justified. The results obtained using this approach can usually be interpreted as rheological models, i.e. as a sequence between springs and stops or skidding blocks.

The paper is organized as follows. The notation used is defined and the mechanical problem is presented in Section 2. The theory of pseudo-friction is outlined, and formal results obtained by performing asymptotic expansions are presented along with the numerical results in Section 3. The numerical implementation of the laws obtained above is carried out in Section 4.

\section{The mechanical problem}

Let us consider a body occupying an open bounded set $\Omega$ of $\mathrm{IR}^{3}$ with a smooth boundary $\partial \Omega$, where the three-dimensional space is referred to the orthonormal frame $\left(O, x_{1}, x_{2}, x_{3}\right)$. This set $\Omega$ is assumed to have a non-empty intersection $S$ with the plane $\left\{x_{3}=0\right\}$. Let $\varepsilon>0$ be a parameter tending to zero, and

$$
\begin{aligned}
& B^{\varepsilon}=\left\{x=\left(x_{1}, x_{2}, x_{3}\right) \in \Omega \text { such that }\left|x_{3}\right|<\frac{\varepsilon}{2}\right\}, \\
& \Omega^{\varepsilon}=\left\{x=\left(x_{1}, x_{2}, x_{3}\right) \in \Omega \text { such that }\left|x_{3}\right|>\frac{\varepsilon}{2}\right\} \\
& \Omega_{ \pm}^{\varepsilon}=\left\{x=\left(x_{1}, x_{2}, x_{3}\right) \in \Omega \text { such that } \pm x_{3}>\frac{\varepsilon}{2}\right\} \\
& S_{ \pm}^{\varepsilon}=\left\{x=\left(x_{1}, x_{2}, x_{3}\right) \in \Omega \text { such that } \pm x_{3}=\frac{\varepsilon}{2}\right\} \\
& \Omega_{ \pm}=\left\{x=\left(x_{1}, x_{2}, x_{3}\right) \in \Omega \text { such that } \pm x_{3}>0\right\} \\
& S=\left\{x=\left(x_{1}, x_{2}, x_{3}\right) \in \Omega \text { such that } x_{3}=0\right\} \\
& \Omega_{0}=\Omega_{+} \cup \Omega_{-}
\end{aligned}
$$

$B^{\varepsilon}$ and $\Omega^{\varepsilon}$ are actually the domains occupied by the adhesive and the adherents, respectively (see Fig. 1 ). The structure is subjected to body force density $\varphi$ and to surface force density $g$ on part $\Gamma_{1}$ of the boundary, whereas it is clamped on the remaining part $\Gamma_{0}$ of the boundary. The two bodies and the joint are assumed to be linearly elastic. We take $\sigma^{\varepsilon}$ and $u^{\varepsilon}$ to denote the stress tensor and the displacement field, respectively, and under the small perturbation hypothesis, the strain tensor is

$$
e_{k h}\left(u^{\varepsilon}\right)=\frac{1}{2}\left(\frac{\partial u_{i}^{\varepsilon}}{\partial x_{j}}+\frac{\partial u_{j}^{\varepsilon}}{\partial x_{i}}\right) .
$$

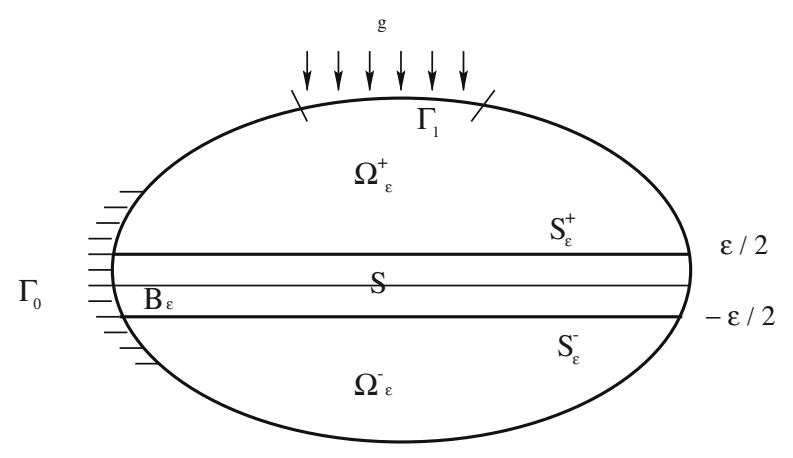

Fig. 1. Geometry of the problem. 
We take $a_{i j k l}$ to denote the elasticity coefficients of the adherents and $\lambda$ and $\mu$ to stand for the Lamé's coefficients of the glue. We have therefore to solve the following problem:

\section{Problem 1}

$$
\left(P_{\varepsilon}\right) \begin{cases}\text { Find }\left(u^{\varepsilon}, \sigma^{\varepsilon}\right) \text { such that : } & \\ \sigma_{i j . j}^{\varepsilon}=-\varphi_{i} & \text { in } \Omega \\ \sigma_{i j}^{\varepsilon}=a_{i j k h} e_{k h}\left(u^{\varepsilon}\right) & \text { in } \Omega^{\varepsilon} \\ \sigma_{i j}^{\varepsilon}=\lambda e_{k k}\left(u^{\varepsilon}\right) \delta_{i j}+2 \mu e_{i j}\left(u^{\varepsilon}\right) & \text { in } B^{\varepsilon} \\ u^{\varepsilon}=0 & \text { on } \Gamma_{0} \\ \sigma^{\varepsilon} n=g & \text { on } \Gamma_{1} \\ + \text { interface laws on } S_{ \pm}^{\varepsilon} & \end{cases}
$$

where $n$ denotes the external unit normal vector to $\Omega$ and interface laws between the bodies and the joint (Signorini's law and Coulomb's law) are defined as follows.

For a given function $\theta: \Omega \mapsto \mathrm{IR}^{3}$, we define the restrictions on $\theta$ on the adherents by $\theta_{\varepsilon}^{ \pm}$and on the glue by $\theta_{\varepsilon}^{m}$. We also define the following jumps of $\theta$

$$
\begin{aligned}
& {[\theta]_{\varepsilon}^{+}:=\theta_{\varepsilon}^{+}\left(x_{1}, x_{2},\left(\frac{\varepsilon}{2}\right)^{+}\right)-\theta_{\varepsilon}^{m}\left(x_{1}, x_{2},\left(\frac{\varepsilon}{2}\right)^{-}\right),} \\
& {[\theta]_{\varepsilon}^{-}:=\theta_{\varepsilon}^{-}\left(x_{1}, x_{2},\left(-\frac{\varepsilon}{2}\right)^{-}\right)-\theta_{\varepsilon}^{m}\left(x_{1}, x_{2},\left(-\frac{\varepsilon}{2}\right)^{+}\right) .}
\end{aligned}
$$

We denote

$$
\begin{aligned}
& \llbracket \theta \rrbracket_{N \varepsilon}^{ \pm}:= \pm[\theta]_{\varepsilon}^{ \pm} e_{3}, \\
& \llbracket \theta \rrbracket_{T \varepsilon}^{ \pm}:=[\theta]_{\varepsilon}^{ \pm}- \pm \llbracket \theta \rrbracket_{N \varepsilon}^{ \pm} e_{3}, \\
& \sigma_{N}^{\varepsilon \pm}:= \pm\left(\sigma^{\varepsilon} e_{3}\right)_{\varepsilon}^{ \pm} e_{3}, \\
& \sigma_{T}^{\varepsilon \pm}:= \pm \sigma^{\varepsilon} e_{3}- \pm \sigma_{N}^{\varepsilon \pm} e_{3} .
\end{aligned}
$$

In Section 3, we assume the contact to involve dry friction conditions between the bodies and the thin layer. The Signorini's law of unilateral contact and the Coulomb's law of dry friction are written in the case of monotonous quasi-static loading (Figs. 2 and 3) as:

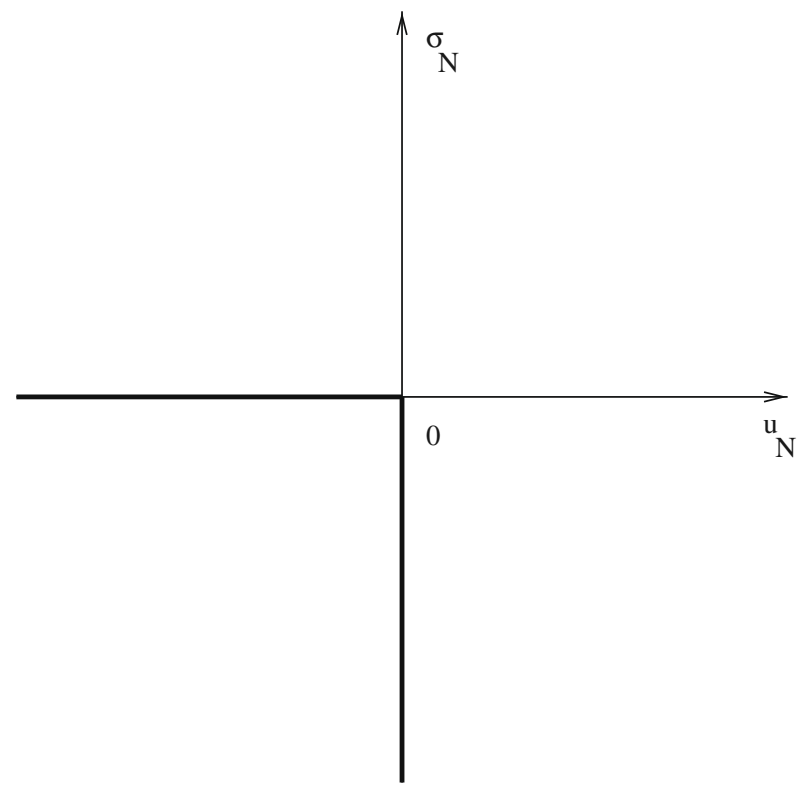

Fig. 2. Unilateral contact 


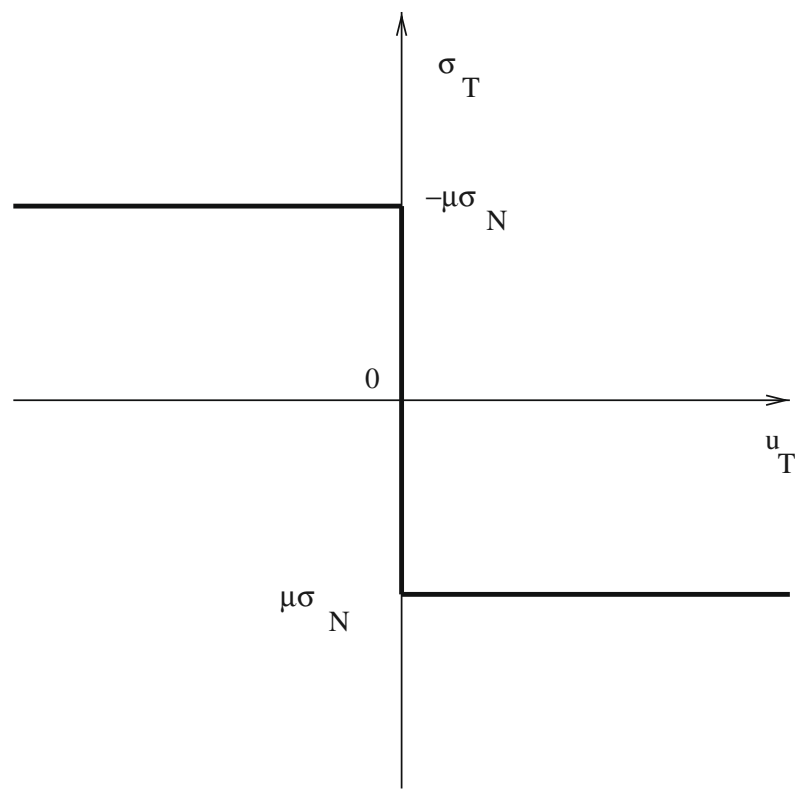

Fig. 3. Dry friction.

$$
\begin{aligned}
& {\left[\sigma^{\varepsilon} e_{3}\right]_{\varepsilon}^{ \pm}=0,} \\
& \sigma_{N}^{\varepsilon \pm} \leqslant 0, \\
& \llbracket u^{\varepsilon} \rrbracket_{N \varepsilon}^{ \pm} \leqslant 0, \\
& \sigma_{N}^{\varepsilon \pm} \llbracket u^{\varepsilon} \rrbracket_{N \varepsilon}^{ \pm}=0, \\
& \left|\sigma_{T}^{\varepsilon \pm}\right| \leqslant f\left|\sigma_{N}^{\varepsilon \pm}\right|, \\
& \text { If }\left|\sigma_{T}^{\varepsilon \pm}\right|<f\left|\sigma_{N}^{\varepsilon \pm}\right| \text { then } \llbracket u^{\varepsilon} \rrbracket_{T \varepsilon}^{ \pm}=0, \\
& \text { If }\left|\sigma_{T}^{\varepsilon \pm}\right|=f\left|\sigma_{N}^{\varepsilon \pm}\right| \text { then } \llbracket u^{\varepsilon} \rrbracket_{T \varepsilon}^{ \pm}=-\zeta \sigma_{T}, \quad \text { with } \zeta \geqslant 0,
\end{aligned}
$$

where $f$ is the friction coefficient.

\section{Frictional contact}

\subsection{Asymptotic expansions}

\subsubsection{General considerations}

The idea underlying matched asymptotic expansions [9,31] is to find two expansions of the displacement $u^{\varepsilon}$ and the stress $\sigma^{\varepsilon}$ in powers of $\varepsilon$, i.e. an external expansion in the case of the bodies and an internal one that for the joint, and to connect these two expansions in order to obtain the same limit. In what follows, we deal with a two-dimensional problem in order to simplify the computations. The relations obtained in the internal expansions we will be expressed using values occurring in the external expansions.

\subsubsection{External expansions}

The external expansion is a classical expansion in powers of $\varepsilon$

$$
\begin{aligned}
& u^{\varepsilon}\left(x_{1}, x_{2}\right)=u^{0}\left(x_{1}, x_{2}\right)+\varepsilon u^{1}\left(x_{1}, x_{2}\right)+\cdots, \\
& e_{i j}\left(u^{\varepsilon}\right)\left(x_{1}, x_{2}\right)=e_{i j}^{0}+\varepsilon e_{i j}^{1}+\cdots, \\
& \left.e_{i j}^{l}=\frac{1}{2} \quad \frac{\partial u_{i}^{l}}{\partial x_{j}}+\frac{\partial u_{j}^{l}}{\partial x_{i}}\right), \\
& \sigma_{i j}^{\varepsilon}\left(x_{1}, x_{2}\right)=\sigma_{i j}^{0}\left(x_{1}, x_{2}\right)+\varepsilon \sigma_{i j}^{1}\left(x_{1}, x_{2}\right)+\cdots
\end{aligned}
$$

\subsubsection{Internal expansions}

In the internal expansions, we perform a blow-up of the second variable. Let $y_{2}=\frac{x_{2}}{\varepsilon}$. The internal expansion gives 


$$
\begin{aligned}
& u^{\varepsilon}\left(x_{1}, x_{2}\right)=v^{0}\left(x_{1}, y_{2}\right)+\varepsilon v^{1}\left(x_{1}, y_{2}\right)+\cdots, \\
& e_{i j}\left(u^{\varepsilon}\right)\left(x_{1}, y_{2}\right)=\varepsilon^{-1} e_{i j}^{-1}+e_{i j}^{0}+\varepsilon e_{i j}^{1}+\cdots, \\
& e_{11}^{l}=\frac{\partial v_{1}^{l}}{\partial x_{1}}, \\
& e_{22}^{l}=\frac{\partial v_{2}^{l+1}}{\partial y_{2}}, \\
& e_{12}^{l}=\frac{1}{2}\left(\frac{\partial v_{2}^{l}}{\partial x_{1}}+\frac{\partial v_{1}^{l+1}}{\partial y_{2}}\right), \\
& \sigma_{i j}^{\varepsilon}\left(x_{1}, y_{2}\right)=\varepsilon^{-1} \tau_{i j}^{-1}\left(x_{1}, y_{2}\right)+\tau_{i j}^{0}\left(x_{1}, y_{2}\right)+\varepsilon \tau_{i j}^{1}\left(x_{1}, y_{2}\right)+\cdots, \\
& \left.\sigma_{i j, j}^{\varepsilon}=\sum_{l=-2}^{\infty} \varepsilon^{l} \frac{\partial \tau_{i 1}^{l}}{\partial x_{1}}+\frac{\partial \tau_{i 2}^{l+1}}{\partial y_{2}}\right) .
\end{aligned}
$$

We use the convention

$$
v^{l}=0, l<0, \quad \tau^{l}=0, \quad l<-1 .
$$

\subsubsection{Continuity conditions}

The third step in this method consists in connecting the two expansions. We chose some intermediate lines defined by $\left.x_{2}= \pm \zeta \varepsilon^{t}, 0<t<1, \zeta \in\right] 0,+\infty\left[\right.$. When $\varepsilon$ tends to zero, $x_{2}$ tends to $0^{ \pm}$and $y_{2}=x_{2} / \varepsilon$ tends to $\pm \infty$. The principle of the method $[9,31]$ consists in assuming that the two expansions give both the same asymptotic limits, that is

$$
\begin{aligned}
& \text { (i) } v^{0}\left(x_{1}, \pm \infty\right)=u^{0}\left(x_{1}, 0^{ \pm}\right), \\
& \text {(ii) } \tau^{-1}\left(x_{1}, \pm \infty\right)=0, \\
& \text { (iii) } \tau^{0}\left(x_{1}, \pm \infty\right)=\sigma^{0}\left(x_{1}, 0^{ \pm}\right) .
\end{aligned}
$$

\subsubsection{Notations}

We introduce some new notations. For a given function $\theta: \Omega_{0} \mapsto R^{d}=1,2$ we define the restrictions on $\theta$ to $\Omega_{ \pm}$by $\theta^{ \pm}$and we also define the following jump of $\theta$ on $S$ as

$$
\llbracket \theta \rrbracket\left(x_{1}\right):=\theta^{+}\left(x_{1}, 0^{+}\right)-\theta^{-}\left(x_{1}, 0^{-}\right) .
$$

We define $C^{ \pm}=\left\{ \pm y_{2} \geqslant 1 / 2\right\}$ and $C^{m}=\left\{\left|y_{2}\right| \leqslant 1 / 2\right\}$. For a given function $\theta\left(x_{1},.\right): \operatorname{IR}^{2} \mapsto \mathbb{R}^{d}, \doteq 1,2$, we define the restrictions on $\theta$ to $C^{ \pm}$by $\theta^{ \pm}$and those to $C^{m}$ by $\theta^{m}$. We also define the following jumps of $\theta$

$$
\begin{aligned}
& {[\theta]_{1 / 2}^{+}\left(x_{1}\right):=\theta^{+}\left(x_{1},\left(\frac{1}{2}\right)^{+}\right)-\theta^{m}\left(x_{1},\left(\frac{1}{2}\right)^{-}\right),} \\
& {[\theta]_{1 / 2}^{-}\left(x_{1}\right):=\theta^{-}\left(x_{1},\left(-\frac{1}{2}\right)^{-}\right)-\theta^{m}\left(x_{1},\left(-\frac{1}{2}\right)^{+}\right) .}
\end{aligned}
$$

We also write

$$
\begin{aligned}
& \llbracket \theta \rrbracket_{N 1 / 2}^{ \pm}:= \pm[\theta]_{1 / 2}^{ \pm} e_{2}, \\
& \llbracket \theta \rrbracket_{T 1 / 2}^{ \pm}:=[\theta]_{1 / 2}^{ \pm}- \pm \llbracket \theta \rrbracket_{N 1 / 2}^{ \pm} e_{2},
\end{aligned}
$$

For a symmetric tensor $\tau$ we define

$$
\begin{aligned}
& \tau_{N}^{ \pm 1 / 2}:= \pm\left(\tau\left(x_{1}, \pm 1 / 2\right) e_{2}\right) e_{2}, \\
& \tau_{T}^{ \pm 1 / 2}:= \pm \tau\left(x_{1}, \pm 1 / 2\right) e_{2}- \pm \tau_{N}^{ \pm 1 / 2} e_{2} .
\end{aligned}
$$

\subsubsection{Elasticity in the thin layer}

Given the constitutive equations in the thin layer, we have

$$
\varepsilon^{-1} \tau_{i j}^{-1}+\tau_{i j}^{0}+\varepsilon \tau_{i j}^{1}+\cdots=\lambda\left(\varepsilon^{-1} e_{k k}^{-1}+e_{k k}^{0}+\varepsilon e_{k k}^{1}+\cdots\right) \delta_{i j}+2 \mu\left(\varepsilon^{-1} e_{i j}^{-1}+e_{i j}^{0}+\varepsilon e_{i j}^{1}+\cdots\right) .
$$

The identification of the various orders depends on the behavior of the Lamé coefficients $\lambda$ and $\mu$ with respect to $\varepsilon$. We obtain nine cases corresponding to the values of the limits $\bar{\lambda}$ and $\bar{\mu}$ of the ratios $\frac{\lambda}{\varepsilon}$ and $\frac{\mu}{\varepsilon}$ :

(a) $\bar{\lambda}=\bar{\mu}=0, \tau_{i j}^{-1}=0, \tau_{i j}^{0}=0$.

(b) $\bar{\lambda}=0,0<\bar{\mu}<\infty, \tau_{i j}^{-1}=0, \tau_{i j}^{0}=2 \bar{\mu} e_{i j}^{-1}$.

(c) $0<\bar{\lambda}<\infty, \bar{\mu}=0, \tau_{i j}^{-1}=0, \tau_{i j}^{0}=\bar{\lambda} e_{k k}^{-1} \delta_{i j}$. 
(d) $0<\bar{\lambda}<\infty, 0<\bar{\mu}<\infty, \tau_{i j}^{-1}=0, \tau_{i j}^{0}=\bar{\lambda} e_{k k}^{-1} \delta_{i j}+2 \bar{\mu} e_{i j}^{-1}$,

(e) $\bar{\lambda}=\infty, \bar{\mu}=0, e_{k k}^{-1}=0, \tau_{i j}^{0}=0$.

(f) $\bar{\lambda}=\infty, 0<\bar{\mu}<\infty, e_{k k}^{-1}=0, \tau_{i j}^{0}=2 \bar{\mu} e_{i j}^{-1}$.

(g) $\bar{\lambda}=0, \bar{\mu}=\infty, e_{i j}^{-1}=0, e_{i j}^{0}=0$.

(h) $0<\bar{\lambda}<\infty, \bar{\mu}=\infty, e_{i j}^{-1}=0, e_{i j}^{0}=0$.

(i) $\bar{\lambda}=\infty, \bar{\mu}=\infty, e_{i j}^{-1}=0, e_{i j}^{0}=0$.

\subsubsection{Contact and friction laws}

Using the usual notations, we will show in what follows that $\tau_{N}^{-1}=0$ and $\tau_{T}^{-1}=0$. In view of the contact and friction laws given by (6), the following conditions are written on the terms of order zero in the asymptotic expansions:

$$
\begin{aligned}
& \llbracket \tau^{0} \rrbracket_{N 1 / 2}^{ \pm}=0, \\
& \left(\tau^{0}\right)_{N}^{ \pm 1 / 2} \leqslant 0, \\
& \llbracket v^{0} \rrbracket_{N 1 / 2}^{ \pm} \leqslant 0, \\
& \left(\tau^{0}\right)_{N}^{ \pm 1 / 2} \llbracket v^{0} \rrbracket_{N 1 / 2}^{ \pm}=0, \\
& \left|\left(\tau^{0}\right)_{T}^{ \pm 1 / 2}\right| \leqslant f\left|\left(\tau^{0}\right)_{N}^{ \pm 1 / 2}\right|, \\
& \text { If }\left|\left(\tau^{0}\right)_{T}^{ \pm 1 / 2}\right|<f\left|\left(\tau^{0}\right)_{N}^{ \pm 1 / 2}\right| \text { then } \llbracket v^{0} \rrbracket_{T 1 / 2}^{ \pm}=0, \\
& \text { If }\left|\left(\tau^{0}\right)_{T}^{ \pm 1 / 2}\right|=f\left|\left(\tau^{0}\right)_{N}^{ \pm 1 / 2}\right| \text { then } \llbracket v^{0} \rrbracket_{T 1 / 2}^{ \pm}=-\zeta\left(\tau^{0}\right)_{T}^{ \pm 1 / 2}, \quad \text { with } \zeta \geqslant 0 .
\end{aligned}
$$

\subsubsection{Equilibrium}

In this paragraph, we consider the equilibrium equation at different orders. We obtain:

\section{Order-2}

$$
\frac{\partial \tau_{i 2}^{-1}}{\partial y_{2}}=0
$$

Hence, from (13ii), we get

$$
\tau_{i 2}^{-1}\left(x_{1}, y_{2}\right)=\tau_{i 2}^{-1}\left(x_{1}, \pm \infty\right)=0 .
$$

From (8), the elasticity law in the bodies gives

$$
\tau_{i 2}^{-1}\left(x_{1}, y_{2}\right)=a_{i 2 j 2} \frac{\partial v_{j}^{0}}{\partial y_{2}}, \quad \tau_{11}^{-1}\left(x_{1}, y_{2}\right)=a_{11 j 1} \frac{\partial v_{j}^{0}}{\partial y_{2}} .
$$

Hence, from (24), we have

$$
\frac{\partial v_{j}^{0}}{\partial y_{2}}=0, \quad v_{j}^{0}\left(x_{1}, y_{2}\right)=v_{j}^{0}\left(x_{1}\right), \quad\left|y_{2}\right|>\frac{1}{2} .
$$

From Eqs. (23) and (24), $\tau_{i j}^{-1}=0$ in the bodies, and, due to the matched conditions

$$
v_{j}^{0}\left(x_{1}, \pm\left|y_{2}\right|\right)=u_{j}^{0}\left(x_{1}, 0^{ \pm}\right) \text {. }
$$

\section{Order-1}

In cases where (a)-(d) $\tau_{1 j}^{-1}$ is equal to zero, we obtain

$$
\frac{\partial \tau_{i 2}^{0}}{\partial y_{2}}=0 .
$$

Using the connecting conditions (13iii), we have

$$
\tau_{i 2}^{0}\left(x_{1}, \pm\left|y_{2}\right|\right)=\sigma_{i 2}^{0}\left(x_{1}, 0\right) .
$$

In cases (e) and (f), we have

$$
\frac{\partial \tau_{22}^{0}}{\partial y_{2}}=0
$$

and, therefore,

$$
\tau_{22}^{0}\left(x_{1}, \pm\left|y_{2}\right|\right)=\sigma_{22}^{0}\left(x_{1}, 0\right) .
$$


In the thin layer, we obtain nine cases corresponding to the behavior of the coefficients. Let $K_{1}=K_{T}=\bar{\mu}$ and $K_{2}=K_{N}=$ $\bar{\lambda}+2 \bar{\mu}$. $f^{+}$is the positive part of a function $f$.

(a) $\bar{\lambda}=\bar{\mu}=0$.In this case, $\tau_{i j}^{0}=0$. Because of the continuity conditions, we obtain

$$
\sigma^{0}\left(x_{1}, 0\right)=0
$$

(b) $\bar{\lambda}=0,0<\bar{\mu}<\infty$. In this case, $\tau_{11}^{0}=0, \tau_{12}^{0}=\bar{\mu} \frac{\partial v_{1}^{0}}{\partial y_{2}}, \tau_{22}^{0}=2 \bar{\mu} \frac{\partial \nu_{2}^{0}}{\partial y_{2}}$.

By integrating and due taking continuity conditions into account, we obtain

$$
\begin{aligned}
& \sigma_{12}^{0}\left(x_{1}, 0\right)=\bar{\mu}\left(v_{1}^{0}\left(x_{1}, \frac{1^{-}}{2}\right)-v_{1}^{0}\left(x_{1},-\frac{1^{+}}{2}\right)\right), \\
& \sigma_{22}^{0}\left(x_{1}, 0\right)=2 \bar{\mu}\left(v_{2}^{0}\left(x_{1}, \frac{1^{-}}{2}\right)-v_{2}^{0}\left(x_{1},-\frac{1^{+}}{2}\right)\right) .
\end{aligned}
$$

First we examine the normal components.

The contact law on surfaces $S_{ \pm}^{\varepsilon}$ gives

$$
\begin{aligned}
& v_{2}^{0}\left(x_{1}, \frac{1^{+}}{2}\right)-v_{2}^{0}\left(x_{1}, \frac{1^{-}}{2}\right) \geqslant 0, \\
& v_{2}^{0}\left(x_{1},-\frac{1^{-}}{2}\right)-v_{2}^{0}\left(x_{1},-\frac{1^{+}}{2}\right) \geqslant 0 .
\end{aligned}
$$

If $\sigma_{22}^{0}\left(x_{1}, 0\right)=0\left(\sigma_{N}^{0}=0\right)$ then (33) and (34) give

$$
\begin{aligned}
& v_{2}^{0}\left(x_{1}, \frac{1^{-}}{2}\right)-v_{2}^{0}\left(x_{1},-\frac{1^{+}}{2}\right)=0, \\
& v_{2}^{0}\left(x_{1}, \frac{1^{+}}{2}\right)-v_{2}^{0}\left(x_{1},-\frac{1^{-}}{2}\right) \geqslant 0,
\end{aligned}
$$

which can be written

$$
\llbracket u^{0} \rrbracket_{N} \leqslant 0 .
$$

If $\sigma_{22}^{0}\left(x_{1}, 0\right)<0\left(\sigma_{N}^{0}<0\right)$ then the contact law and (33) give

$$
\begin{aligned}
& v_{2}^{0}\left(x_{1}, \frac{1^{+}}{2}\right)-v_{2}^{0}\left(x_{1}, \frac{1^{-}}{2}\right)=0, \\
& v_{2}^{0}\left(x_{1},-\frac{1^{-}}{2}\right)-v_{2}^{0}\left(x_{1},-\frac{1^{+}}{2}\right)=0, \\
& \sigma_{22}^{0}\left(x_{1}, 0\right)=2 \bar{\mu}\left(v_{2}^{0}\left(x_{1}, \frac{1^{+}}{2}\right)-v_{2}^{0}\left(x_{1},-\frac{1^{-}}{2}\right)\right),
\end{aligned}
$$

which can be written

$$
\sigma_{N}^{0}\left(x_{1}, 0\right)=-2 \bar{\mu}\left[\left[u^{0}\right]\right]_{N} .
$$

We then examine the tangential components.

If $\left|\sigma_{12}^{0}\right|<f\left|\sigma_{22}^{0}\right|\left(\left|\sigma_{T}^{0}\right|<f\left|\sigma_{N}^{0}\right|\right)$ then the friction law and (33) give

$$
\begin{aligned}
& v_{1}^{0}\left(x_{1}, \frac{1^{+}}{2}\right)-v_{1}^{0}\left(x_{1}, \frac{1^{-}}{2}\right)=0, \\
& v_{1}^{0}\left(x_{1},-\frac{1^{-}}{2}\right)-v_{1}^{0}\left(x_{1},-\frac{1^{+}}{2}\right)=0, \\
& \sigma_{12}^{0}\left(x_{1}, 0\right)=\bar{\mu}\left(v_{1}^{0}\left(x_{1}, \frac{1^{+}}{2}\right)-v_{1}^{0}\left(x_{1},-\frac{1^{-}}{2}\right)\right),
\end{aligned}
$$

which can be written

$$
\sigma_{T}^{0}\left(x_{1}, 0\right)=-\bar{\mu}\left[\left[u^{0}\right]\right]_{T} .
$$


If $\left|\sigma_{12}^{0}\right|=f\left|\sigma_{22}^{0}\right|\left(\left|\sigma_{T}^{0}\right|=f\left|\sigma_{N}^{0}\right|\right)$ then the friction law and (24) give

$$
\begin{aligned}
& v_{1}^{0}\left(x_{1}, \frac{1^{+}}{2}\right)-v_{1}^{0}\left(x_{1}, \frac{1^{-}}{2}\right)=\zeta_{1} \sigma_{12}^{0}\left(x_{1}, 0\right), \\
& v_{1}^{0}\left(x_{1},-\frac{1^{-}}{2}\right)-v_{1}^{0}\left(x_{1},-\frac{1^{+}}{2}\right)=\zeta_{2} \sigma_{12}^{0}\left(x_{1}, 0\right), \\
& v_{1}^{0}\left(x_{1}, \frac{1^{+}}{2}\right)-v_{1}^{0}\left(x_{1},-\frac{1^{-}}{2}\right)=\left(\frac{1}{\bar{\mu}}+\zeta_{1}+\zeta_{2}\right) \sigma_{12}^{0}\left(x_{1}, 0\right),
\end{aligned}
$$

which can be written

$$
\left[\left[u^{0}\right]\right]_{T}=-\left(\frac{1}{\bar{\mu}}+\zeta\right) \sigma_{T}^{0}\left(x_{1}, 0\right) .
$$

To summarize, we obtain

$$
\begin{aligned}
& \sigma_{N}^{0}=-2 \bar{\mu} \llbracket u^{0} \rrbracket_{N}^{+}, \\
& \left|\sigma_{T}^{0}\right| \leqslant f\left|\sigma_{N}^{0}\right|, \\
& \text { If }\left|\sigma_{T}^{0}\right|<f\left|\sigma_{N}^{0}\right| \text { then } \sigma_{T}^{0}=-\bar{\mu} \llbracket u^{0} \rrbracket_{T}, \\
& \text { If }\left|\sigma_{T}^{0}\right|=f\left|\sigma_{N}^{0}\right| \text { then } \llbracket u^{0} \rrbracket_{T}=-\zeta \sigma_{T}^{0}, \quad \zeta \geqslant 0 .
\end{aligned}
$$

(c) $0<\bar{\lambda}<\infty, \bar{\mu}=0$.

In this case $\tau_{11}^{0}=\tau_{22}^{0}=\bar{\lambda} \frac{\partial v_{2}^{0}}{\partial y_{2}} \tau_{12}^{0}=0$. For the normal part, we proceed as in (b), by replacing $2 \bar{\mu}$ by $\bar{\lambda}$. The tangential part corresponds to (a). We obtain

$$
\begin{aligned}
& \sigma_{N}^{0}=-\bar{\lambda} \llbracket u^{0} \rrbracket_{N}^{+}, \\
& \sigma_{T}^{0}=0 .
\end{aligned}
$$

(d) $0<\bar{\lambda}<\infty, 0<\bar{\mu}<\infty$.

In this case, $\tau_{11}^{0}=\bar{\lambda} \frac{\partial v_{2}^{0}}{\partial y_{2}}, \tau_{i 2}^{0}=K_{i} \frac{\partial v_{i}^{0}}{\partial y_{2}}$. Here, we again proceed as in (b). We obtain

$\sigma_{N}^{0}=-(\bar{\lambda}+2 \bar{\mu}) \llbracket u^{0} \rrbracket_{N}^{+}$,

$\left|\sigma_{T}^{0}\right| \leqslant f\left|\sigma_{N}^{0}\right|$,

If $\left|\sigma_{T}^{0}\right|<f\left|\sigma_{N}^{0}\right| \quad$ then $\sigma_{T}^{0}=-\bar{\mu} \llbracket u^{0} \rrbracket_{T}$,

If $\left|\sigma_{T}^{0}\right|=f\left|\sigma_{N}^{0}\right| \quad$ then $\llbracket u_{\rrbracket}^{0} T=-\zeta \sigma_{T}^{0}, \quad \zeta \geqslant 0$.

(e) $\bar{\lambda}=\infty, \bar{\mu}=0$.

We have $\frac{\partial v_{2}^{0}}{\partial y_{2}}=0, \tau_{12}^{0}=0$. For the normal tangential part, we proceed as in (a). The normal part becomes

$v_{2}^{0}\left(x_{1}, \frac{1^{-}}{2}\right)=v_{2}^{0}\left(x_{1},-\frac{1^{+}}{2}\right)$.

If $\sigma_{22}^{0}<0\left(\sigma_{N}<0\right)$ then

$$
\begin{aligned}
& v_{2}^{0}\left(x_{1}, \frac{1^{+}}{2}\right)=v_{2}^{0}\left(x_{1},-\frac{1^{-}}{2}\right), \\
& \llbracket u^{0} \rrbracket_{N}=0 . \\
& \text { If } \sigma_{22}^{0}=0\left(\sigma_{N}=0\right), \text { we have } \\
& v_{2}^{0}\left(x_{1}, \frac{1^{+}}{2}\right)-v_{2}^{0}\left(x_{1},-\frac{1^{-}}{2}\right) \geqslant 0, \\
& \llbracket u^{0} \rrbracket_{N} \leqslant 0 .
\end{aligned}
$$

To summarize, we have

$$
\begin{aligned}
& \llbracket u^{0} \rrbracket_{N} \leqslant 0, \sigma_{N}^{0} \leqslant 0, \llbracket u^{0} \rrbracket_{N} \sigma_{N}^{0}=0, \\
& \sigma_{T}^{0}=0 .
\end{aligned}
$$

(f) $\bar{\lambda}=\infty, 0<\bar{\mu}<\infty$.

We have $\frac{\partial \nu_{2}^{0}}{\partial y_{2}}=0, \tau_{12}^{0}=\bar{\mu} \frac{\partial v_{1}^{0}}{\partial y_{2}}$. The treatment of the tangential part is similar to (b) and the treatment of the normal part is similar to (e). To summarize, we obtain 


$$
\begin{aligned}
& \llbracket u^{0} \rrbracket_{N} \leqslant 0, \quad \sigma_{N}^{0} \leqslant 0, \quad \llbracket u^{0} \rrbracket_{N} \sigma_{N}^{0}=0, \\
& \left|\sigma_{T}^{0}\right| \leqslant f\left|\sigma_{N}^{0}\right|, \\
& \text { If }\left|\sigma_{T}^{0}\right|<f\left|\sigma_{N}^{0}\right| \quad \text { then } \sigma_{T}^{0}=-\bar{\mu} \llbracket u^{0} \rrbracket_{T}, \\
& \text { If }\left|\sigma_{T}^{0}\right|=f\left|\sigma_{N}^{0}\right| \quad \text { then } \llbracket u^{0} \rrbracket_{T}=-\zeta \sigma_{T}^{0}, \quad \zeta \geqslant 0 .
\end{aligned}
$$

The three last cases call for special treatment.

(g)(h)(i) $\bar{\mu}=\infty$.

In these cases, we have $\frac{\partial v_{i}^{0}}{\partial y_{2}}=0$. We proceed as in (e) on the normal and tangential parts. This gives

$$
v_{i}^{0}\left(x_{1}, \frac{1^{-}}{2}\right)=v_{i}^{0}\left(x_{1},-\frac{1^{+}}{2}\right)
$$

Here, we proceed as in the previous cases.

If $\sigma_{22}^{0}<0\left(\sigma_{N}<0\right)$, then

$$
\begin{gathered}
v_{2}^{0}\left(x_{1}, \frac{1^{+}}{2}\right)=v_{2}^{0}\left(x_{1},-\frac{1^{-}}{2}\right), \\
\text { and thus } \llbracket u^{0} \rrbracket_{N}=0 .
\end{gathered}
$$

If $\sigma_{22}^{0}=0\left(\sigma_{N}=0\right)$, as in the previous paragraph,

$$
\begin{aligned}
& v_{2}^{0}\left(x_{1}, \frac{1^{+}}{2}\right)-v_{2}^{0}\left(x_{1},-\frac{1^{-}}{2}\right) \geqslant 0, \\
& \text { thus } \llbracket u^{0} \rrbracket_{N} \leqslant 0 .
\end{aligned}
$$

If $\left|\sigma_{12}^{0}\right|<f\left|\sigma_{22}^{0}\right|\left(\left|\sigma_{T}^{0}\right|<f\left|\sigma_{N}^{0}\right|\right)$, then we have

$$
\begin{aligned}
& v_{1}^{0}\left(x_{1}, \frac{1^{+}}{2}\right)-v_{1}^{0}\left(x_{1}, \frac{1^{-}}{2}\right)=0, \\
& v_{1}^{0}\left(x_{1},-\frac{1^{-}}{2}\right)-v_{1}^{0}\left(x_{1},-\frac{1^{+}}{2}\right)=0, \\
& \text { and hence } \llbracket u^{0} \rrbracket_{T}=0 .
\end{aligned}
$$

If $\left|\sigma_{12}^{0}\right|=f\left|\sigma_{22}^{0}\right|\left(\left|\sigma_{T}^{0}\right|=f\left|\sigma_{N}^{0}\right|\right)$, then the friction law and (24) gives

$$
\begin{aligned}
& v_{1}^{0}\left(x_{1}, \frac{1^{+}}{2}\right)-v_{1}^{0}\left(x_{1}, \frac{1^{-}}{2}\right)=\zeta_{1} \sigma_{12}^{0}\left(x_{1}, 0\right), \\
& v_{1}^{0}\left(x_{1},-\frac{1^{-}}{2}\right)-v_{1}^{0}\left(x_{1},-\frac{1^{+}}{2}\right)=\zeta_{2} \sigma_{12}^{0}\left(x_{1}, 0\right), \\
& v_{1}^{0}\left(x_{1}, \frac{1^{+}}{2}\right)-v_{1}^{0}\left(x_{1},-\frac{1^{-}}{2}\right)=\left(\zeta_{1}+\zeta_{2}\right) \sigma_{12}^{0}\left(x_{1}, 0\right), \\
& \llbracket u^{0} \rrbracket_{T}=-\zeta \sigma_{T}^{0}\left(x_{1}, 0\right) .
\end{aligned}
$$

Therefore,

$$
\begin{aligned}
& \llbracket u^{0} \rrbracket_{N} \leqslant 0, \quad \sigma_{N}^{0} \leqslant 0, \quad \llbracket u^{0} \rrbracket_{N} \sigma_{N}^{0}=0, \\
& \left|\sigma_{T}^{0}\right| \leqslant f\left|\sigma_{N}^{0}\right|, \\
& \text { If }\left|\sigma_{T}^{0}\right|<f\left|\sigma_{N}^{0}\right| \quad \text { then } \llbracket u^{0} \rrbracket_{T}=0, \\
& \text { If }\left|\sigma_{T}^{0}\right|=f\left|\sigma_{N}^{0}\right| \quad \text { then } \llbracket u^{0} \rrbracket_{T}=-\zeta \sigma_{T}^{0}, \quad \zeta \geqslant 0 .
\end{aligned}
$$

The nine cases are summarized in Table 1.

\subsection{Numerical results}

In this part, we proceed as previous studies [28,20]. The example of a dovetail assembly (Figs. 4 and 5) is treated [29]. The aim of this numerical study is to confirm that the numerical results are coherent with the theory (validation) and to determine the thickness of the layer at which the limit interface law can be taken to be valid (quantification). The initial problem is therefore solved numerically in the case of a thin layer with decreasing thickness and stiffness values, as done by OuldKhaoua et al. [28]. A relaxation procedure is used for this purpose, as in previous studies [13,29].

The numerical results obtained are then compared with the theoretical results. In order to simplify the computations, the Lamé's coefficients of the joint are assumed to be given by 
Table 1

Interface laws with frictional contact (with 1: $\left|\sigma_{T}\right|<f\left|\sigma_{N}\right|, 2:\left|\sigma_{T}\right|=f\left|\sigma_{N}\right|$ ).

\begin{tabular}{|c|c|c|c|}
\hline$\mu / \varepsilon \rightarrow 0$ & $\llbracket u \rrbracket_{N} \leqslant 0, \sigma_{N} \leqslant 0, \llbracket u \rrbracket_{N} \sigma_{N}=0, \sigma_{T}=0$ & $\sigma_{N}=-\bar{\lambda} \llbracket u \rrbracket_{N}^{+}, \sigma_{T}=0$ & $\sigma_{N}=0, \sigma_{T}=0$ \\
\hline$\mu / \varepsilon \rightarrow \bar{\mu}$ & $\begin{array}{l}\llbracket u \rrbracket_{N} \leqslant 0, \sigma_{N} \leqslant 0, \llbracket u \rrbracket_{N} \sigma_{N}=0,\left|\sigma_{T}\right| \leqslant f\left|\sigma_{N}\right| \\
\text { if } 1 \Rightarrow \sigma_{T}=-\bar{\mu} \llbracket u \rrbracket_{T}, \text { if } 2 \Rightarrow \llbracket u \rrbracket_{T}=-\zeta \sigma_{T}\end{array}$ & $\begin{array}{l}\sigma_{N}=-(\bar{\lambda}+2 \bar{\mu}) \llbracket u \rrbracket_{N}^{+},\left|\sigma_{T}\right| \leqslant f\left|\sigma_{N}\right| \\
\text { if } 1 \Rightarrow \sigma_{T}=-\bar{\mu} \llbracket u \rrbracket_{T}, \text { if } 2 \Rightarrow \llbracket u \rrbracket_{T}=-\zeta \sigma_{T}\end{array}$ & $\begin{array}{l}\sigma_{N}=-2 \bar{\mu} \llbracket u \rrbracket_{N}^{+},\left|\sigma_{T}\right| \leqslant f\left|\sigma_{N}\right|, \text { if } 1 \Rightarrow \sigma_{T}=-\bar{\mu} \\
\llbracket u) \rrbracket_{T}, \text { if } 2 \Rightarrow \llbracket u \rrbracket_{T}=-\zeta \sigma_{T}\end{array}$ \\
\hline$\mu / \varepsilon \rightarrow \infty$ & $\begin{array}{l}\llbracket u \rrbracket_{N} \leqslant 0, \sigma_{N} \leqslant 0, \llbracket u \rrbracket_{N} \sigma_{N}=0,\left|\sigma_{T}\right| \leqslant f\left|\sigma_{N}\right| \\
\text { if } 1 \Rightarrow \llbracket u \rrbracket_{T}=0, \text { if } 2 \Rightarrow \llbracket u \rrbracket_{T}=-\zeta \sigma_{T} \\
\lambda / \varepsilon \rightarrow \infty\end{array}$ & $\begin{array}{l}\llbracket u \rrbracket_{N} \leqslant 0, \sigma_{N} \leqslant 0, \llbracket u \rrbracket_{N} \sigma_{N}=0,\left|\sigma_{T}\right| \leqslant f\left|\sigma_{N}\right| \\
\text { if } 1 \Rightarrow \llbracket u \rrbracket_{T}=0, \text { if } 2 \Rightarrow \llbracket u \rrbracket_{T}=-\zeta \sigma_{T} \\
\lambda / \varepsilon \rightarrow \bar{\lambda}\end{array}$ & $\begin{array}{l}\llbracket u \rrbracket_{N} \leqslant 0, \sigma_{N} \leqslant 0, \llbracket u \rrbracket_{N} \sigma_{N}=0,\left|\sigma_{T}\right| \leqslant f\left|\sigma_{N}\right|, \\
\text { if } 1 \Rightarrow \llbracket u \rrbracket_{T}=0 \text {, if } 2 \Rightarrow \llbracket u \rrbracket_{T}=-\zeta \sigma_{T} \\
\lambda / \varepsilon \rightarrow 0\end{array}$ \\
\hline
\end{tabular}

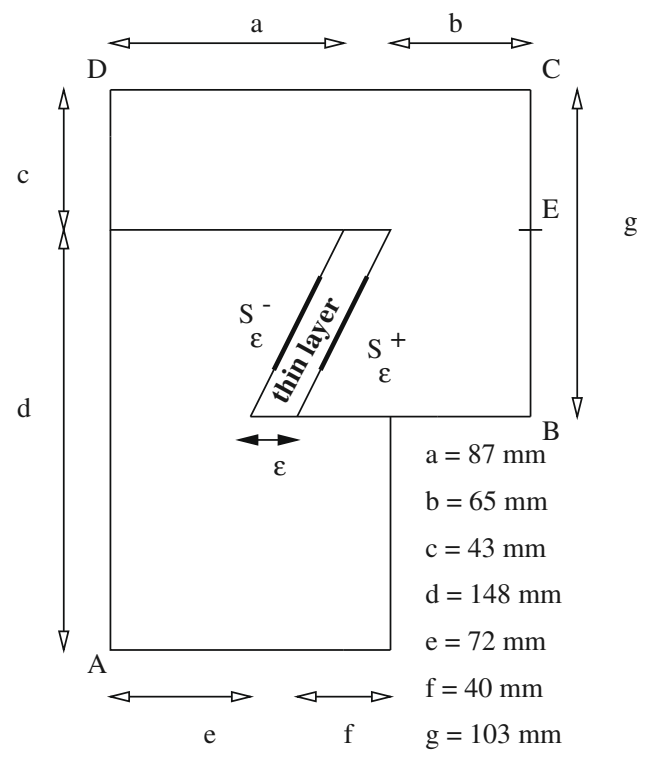

Fig. 4. The dovetail assembly.

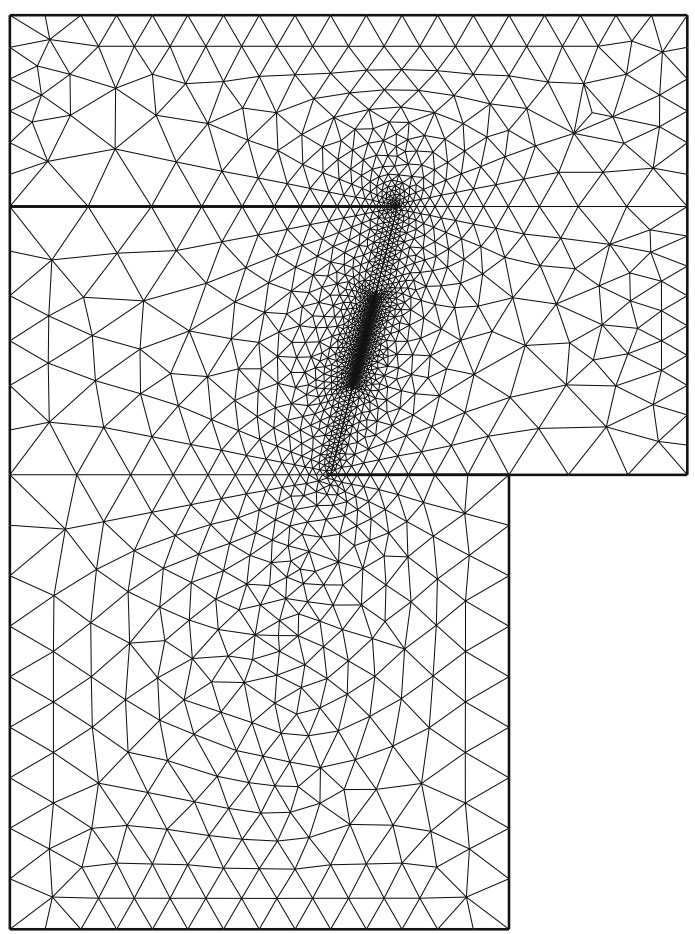

Fig. 5. Mesh of the structure. 
Table 2

The nine cases.

Case (e) $\gamma=0.5, \delta=1.5$

Case (f) $\gamma=0.5, \delta=1$

Case (i) $\gamma=0.5, \delta=0.5$
Case (c) $\gamma=1, \delta=1.5$

Case (d) $\gamma=1, \delta=1$

Case (h) $\gamma=1, \delta=0.5$
Case (a) $\gamma=1.5, \delta=1.5$

Case (b) $\gamma=1.5, \delta=1$

Case (g) $\gamma=1.5, \delta=0.5$

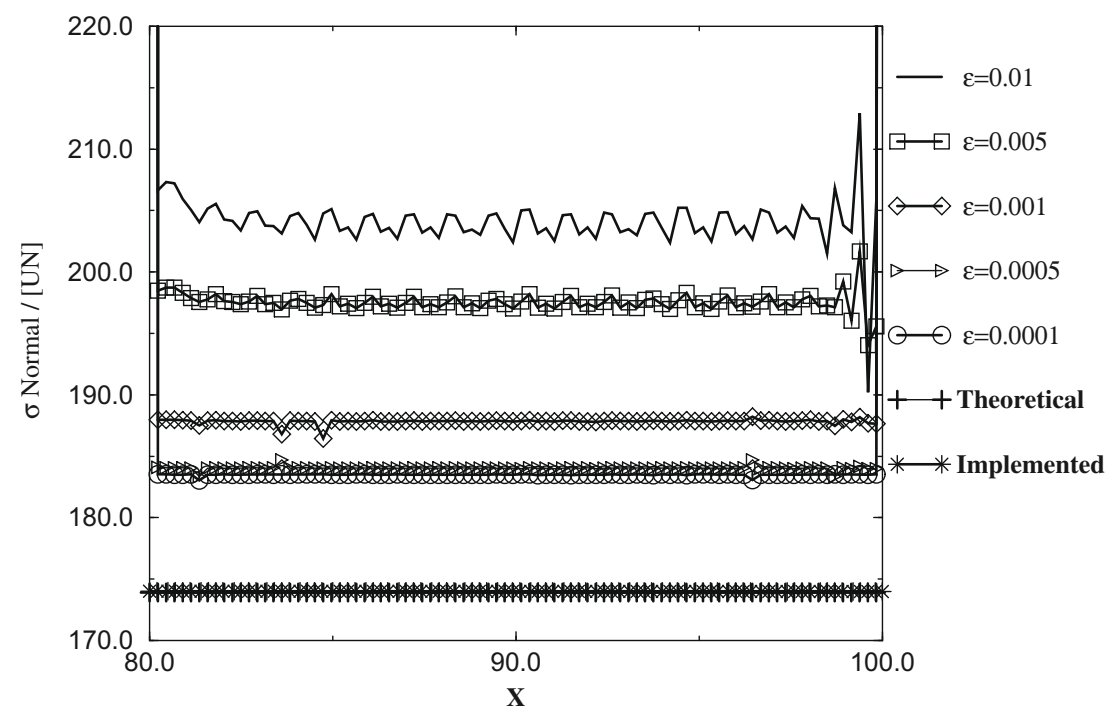

Fig. 6. Ratio between normal stress and normal displacement (case b).

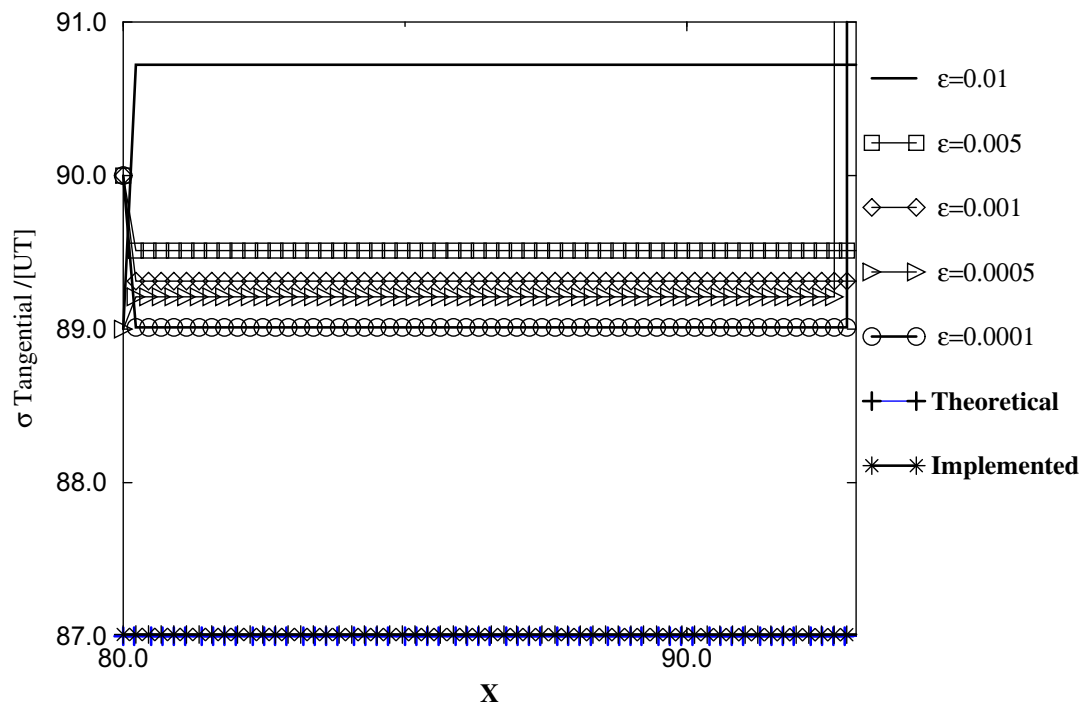

Fig. 7. Ratio between tangential stress and tangential displacement (case d).

$$
\begin{cases}\lambda_{\varepsilon}=\varepsilon^{\gamma} \lambda, & \gamma \geqslant 0 \\ \mu_{\varepsilon}=\varepsilon^{\delta} \mu, & 0 \leqslant \delta<2 .\end{cases}
$$

The problem is solved with decreasing values of $\varepsilon$,

$$
1 \times 10^{-2}, 5 \times 10^{-3}, 1 \times 10^{-3}, 5 \times 10^{-4}, 1 \times 10^{-4} .
$$

In order to simplify the procedure, the notations given in Table 2 are used. 


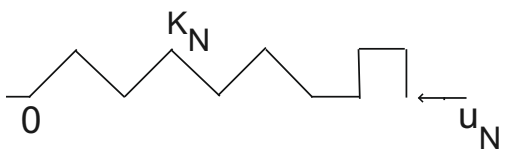

Fig. 8. Interpretation of the normal part of the limit law.

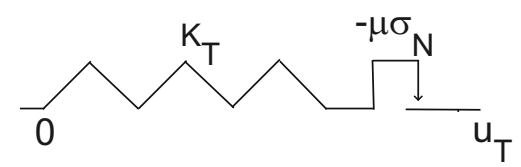

Fig. 9. Interpretation of the tangential part of the limit law.

In order to analyze the results obtained, the displacement fields $\left(u_{N}, u_{T}\right)$ and the stress vector $\left(\sigma_{N}, \sigma_{T}\right)$ were computed on $S_{\varepsilon}^{ \pm}$in the case of the initial problem (and on $S$ in that of the limit problem) in the nine cases studied. The theoretical and numerical curves of the stick and sliding nodes are then compared. The friction problem is solved by means of a fixed point algorithm coupled with a relaxation procedure [29]. Similar results were obtained (see Figs. 6 and 7). As can be seen from these figures, the limit law can be taken to be valid for at thin layer thickness up to $10^{-3} \mathrm{~mm}$. This value is suitable for a large class of problems (for example in glue-bonding processes).

\subsection{Predictive nature of the model}

In this section, we focus on a particular characteristic of the model, its predictive nature which depends on the mechanical and geometrical parameters of the glue and the adherents. The model presented in the previous paragraphs involves four parameters: the thickness of the glue $\varepsilon$, the stiffness coefficients of the glue $\lambda$ and $\mu$ and the friction coefficient $f$.

We observe in Table 1, that the various cases depend both on the limits of $\lambda / \varepsilon$ and of $\mu / \varepsilon$. We stress the fact that the nine laws that appear in Table 1 depend only on the relative values of the parameters. If mathematically, these limits are perfectly defined, mechanically, in a non academic problem, they are a priori unknown. The engineer only knows (or has the possibility of knowing) the thickness and the stiffness of the glue. The limits are approximated in a satisfactory way by the ratio between the stiffness and the thickness. In other words, in Table 1 , the value of $\bar{\lambda}$ and $\bar{\mu}$ can be replaced by $\lambda / \varepsilon$ and $\mu / \varepsilon$, respectively. But these approximations are never equal to zero or to infinity. So when shall we consider that these limit values are obtained? Simply by comparison to a reference value. For example in the case of isotropic adherents, the reference values, would be $\lambda_{m} / L$ or $\mu_{m} / L$, where $\lambda_{m}$ and $\mu_{m}$ are the stiffness coefficients and $L$ the characteristic length of the adherents (chosen equal to 1 in the numerical applications), with $\lambda \ll \lambda_{m}, \mu \ll \mu_{m}$ and $\varepsilon \ll L$, which expresses the fact that the interface is both soft and thin.

More precisely, when

- $\lambda / \varepsilon$ and $\mu / \varepsilon$ are very small by comparison to the reference value, i.e. the glue is relatively more soft than thin, $\bar{\lambda}$ and $\bar{\mu}$ shall be taken equal to zero;

- $\lambda / \varepsilon$ and $\mu / \varepsilon$ are very large by comparison to the reference value, i.e. the glue is relatively more thin than soft, $\bar{\lambda}$ and $\bar{\mu}$ shall be taken equal to infinity;

- $\lambda / \varepsilon$ and $\mu / \varepsilon$ are finite by comparison to the reference value, i.e. the glue is as thin as soft, $\bar{\lambda}$ and $\bar{\mu}$ shall be taken equal to $\lambda / \varepsilon$ and $\mu / \varepsilon$, respectively.

Numerically, we can observe that "very small" corresponds to less than $3 \%$ of the reference value and that "very large" corresponds to more than 30 times the reference value. The "finite case" corresponds to the range between these two extreme values.

\subsection{Rheological interpretation}

Based on the mechanical model obtained above, this model can be interpreted in rheological terms. The normal part of the model (contact law) can be interpreted as the sequence between a spring with stiffness $K_{N}$ and a stop (Fig. 8). Elastic behavior is classically modeled using spring and unilateral contact using a stop. At the limit, the behavior of the thin layer is conserved: the spring corresponds to the elastic part of the model, and the stop corresponds to the non-linear part. In fact, at the limit, the elasticity-unilateral contact sequence becomes a spring-stop sequence. This result is quite general. As an example, an interface consisting of two thin layers with different stiffnesses will become a sequence of two springs with different rigidities. On similar lines, the tangential part of the present model can be regarded as a sequence between a spring corresponding to the elasticity of the thin layer and a skidding block corresponding to the friction on the surface between the adherents and the adhesive (Fig. 9). 


\section{Numerical implementation}

In what follows, we will adopt the two-dimensional context, focusing on the contact between a deformable solid $A$ occupying $\Omega$ and a rigid body. In this case,

\section{Problem 2}

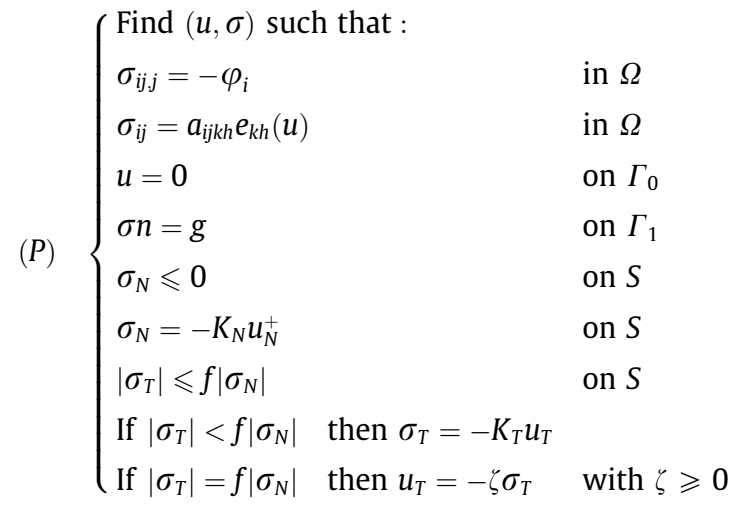

The contact laws are shown in Figs. 10 and 11. In lines with [8], the above problem is equivalent to:

Problem 3. Find $\rho$, the fixed point of the application $\rho \rightarrow f\left|\sigma_{N}(u(\rho))\right|$, where $u=u(\rho)$ is the solution of $\left\{\begin{array}{l}\text { Find } u \in V \text { such that : } \\ J(u) \leqslant J(v), \quad \forall v \in V\end{array}\right.$

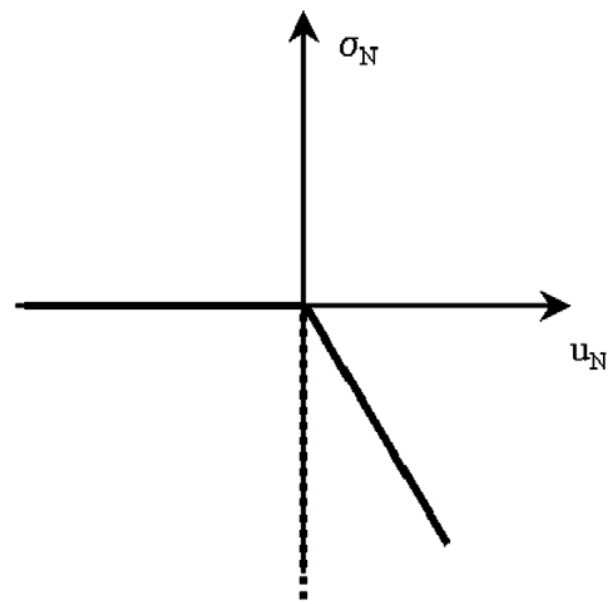

Fig. 10. Contact law: normal component (the dotted line corresponds to an infinite value of $K_{N}$ ).

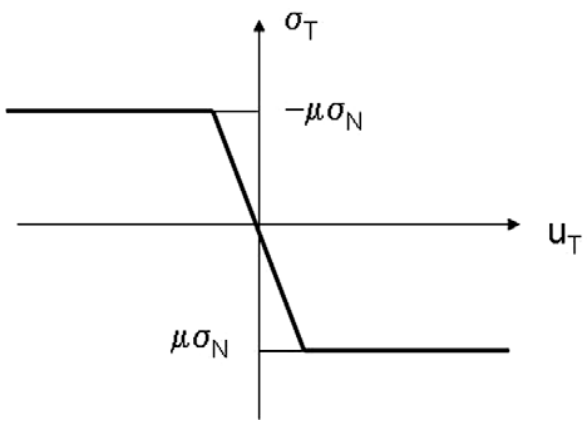

Fig. 11. Contact law: tangential component (the dotted line corresponds to an infinite value of $K_{T}$ ). 
with

$$
J(v)=\frac{1}{2} \int_{\Omega} a e(v) e(v) d x-\int_{\Omega} \varphi \cdot u d x+\int_{\Gamma_{1}} g \cdot u d l+\int_{S} \phi\left(v_{N}\right) d s+\int_{S} \psi\left(v_{T}\right) d s
$$

where $\phi$ is given by

$$
\phi\left(v_{N}\right)=\frac{K_{N}}{2}\left(v_{N}^{+}\right)^{2} .
$$

and $\psi$ is given by

$$
\psi\left(v_{T}\right)=\frac{1}{2} K_{T} \cdot\left(v_{T}\right)^{2}+\rho\left(\left|v_{T}\right|-\frac{\rho}{K_{T}}\right)^{+} .
$$

Problem 3 is discretized using a finite element method formulated in terms of the displacements. We usually adopt $P 1$ finite elements (triangles with three nodes and six degrees of freedom) or $Q 1$ finite elements (quadrangles with four nodes and eight degrees of freedom). We have to minimize a functional still denoted $J$ for $v$ in $R^{2 N P}$ such that

$$
\mathscr{J}(v)=\frac{1}{2} v^{T} A v+\frac{1}{2}(P v)^{T} B(P v)-v^{T} L
$$

The following notations are used:

NP: total number of nodes,

$\mathrm{NC}$ : number of contact nodes,

$I_{N}$ : indices of normal components of contact nodes,

$I_{T}$ : indices of tangential components of contact nodes,

$A$ : stiffness matrix associated with volume terms with coefficient $a_{i j}$,

$B$ : stiffness matrix to the surface terms with coefficient $b_{i j}$,

$P$ : projection from $R^{2 N P}$ to $R^{2 N C}$,

$L$ : generalized loading vector with coefficients $L_{i}$.

\subsection{Relaxation method with constraints} in $R^{2 N P}$

The relaxation method [13] consists in finding the solution to Problem 2 by solving a sequence of minimization problems

$$
\left\{\begin{array}{l}
\text { Find } u_{i}^{n+\frac{1}{2}} \text { such that } \forall v \in R^{2 N P} \\
\mathscr{J}\left(u_{1}^{n+1}, \ldots, u_{i-1}^{n+1}, u_{i}^{n+\frac{1}{2}}, u_{i+1}^{n}, \ldots, u_{2 N P}^{n}\right) \leqslant \mathscr{J}\left(u_{1}^{n+1}, \ldots, u_{i-1}^{n+1}, v, u_{i+1}^{n}, \ldots, u_{2 N P}^{n}\right),
\end{array}\right.
$$

$\omega$ is taken to denote the relaxation coefficient.

First we deal with the normal components.

- $i \in I_{N}$

The algorithm is written

$$
\begin{aligned}
& \left(u_{i}^{n+\frac{1}{2}}=\frac{1}{d_{i i}^{n+\frac{1}{2}}} \quad L_{i}-\sum_{j=1}^{i-1} d_{i j}^{n+1} u_{j}^{n+1}-\sum_{j=i+1}^{2 N P} d_{i j}^{n} u_{j}^{n}\right) \\
& \text { with } \\
& d_{i j}^{n}= \begin{cases}a_{i j}+\gamma\left(u_{j}^{n}\right) b_{i j} & \text { if } j \in I_{N} \\
a_{i j}+\eta\left(u_{j}^{n}\right) b_{i j} & \text { if } j \in I_{T} \\
a_{i j} & \text { otherwise }\end{cases} \\
& \gamma(u)= \begin{cases}0 & \text { if } u \leqslant 0 \\
1 & \text { if } u>0\end{cases} \\
& \text { and } \eta(u)= \begin{cases}0 & \text { if }|u|>\frac{\rho}{K_{T}} \\
1 & \text { if }|u| \leqslant \frac{\rho}{K_{T}}\end{cases} \\
& u_{i}^{n+1}=(1-\omega) u_{i}^{n}+\omega u_{i}^{n+\frac{1}{2}}
\end{aligned}
$$


As regards the tangential components, we have

- $i \in I_{T}$

First we take the fixed point problem $\rho_{i}^{l+1}=f\left|\sigma_{N}\left(u\left(\rho_{i}^{l}\right)\right)\right|$ and write

$$
\left\{\begin{array}{l}
u_{i}^{n+\frac{1}{2}}=\frac{1}{d_{i i}^{n+\frac{1}{2}}}\left(L_{i}-\sum_{j=1}^{i-1} d_{i j}^{n+1} u_{j}^{n+1}\right. \\
\text { with } \\
\theta(u)= \begin{cases}-1 & \text { if } u<\frac{-\rho}{K_{T}} \\
1 & \text { if } u>\frac{\rho}{K_{T}} \\
0 & \text { if }|u| \leqslant \frac{\rho}{K_{T}}\end{cases} \\
u_{i}^{n+1}=(1-\omega) u_{i}^{n}+\omega u_{i}^{n+\frac{1}{2}}
\end{array}\right.
$$

\subsection{Comments}

This algorithm is accelerated by means of a diagonal procedure consisting partly in partially solving the problem with a given $\rho^{l}$ [29]. Another means of improvement consists in applying condensation method to the contact nodes in order to shorten the procedure [21]. The above algorithm can easily be extended to the contact between several deformable bodies.

\subsection{Testing the validity of the algorithm: compression of a bar}

In this paragraph the algorithm is tested and its validity is confirmed. We used a benchmark test developed by the group working on "Validation of computer codes" at the French Research Group "Large Deformations and Damage" [29].

Here we adopt the context of plane strains and take the case of a long bar with a square section (Fig. 12) and Lame's coefficients $\lambda=45 \mathrm{GPa}$ and $\mu=54 \mathrm{GPa}$.

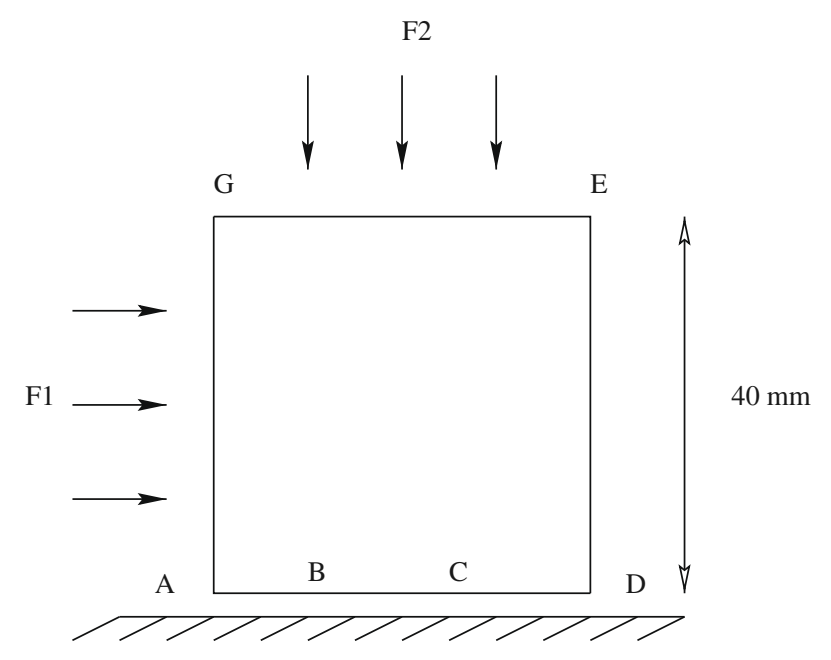

Fig. 12. The problem of the long bar.

\section{Table 3}

Behavior of the interface for some stiffness.

\begin{tabular}{|c|c|c|c|}
\hline Stiffness & Gap AB & Sliding $\left|\sigma_{T}\right|=f\left|\sigma_{N}\right| \mathrm{BC}$ & Stick $\left|\sigma_{T}\right|<f\left|\sigma_{N}\right| \mathrm{CD}$ \\
\hline (1) $\bar{\mu}=5.4 \times 10^{+8} ; \bar{\lambda}=4.5 \times 10^{+8}$ & 3 nodes & 16 nodes & 13 nodes \\
\hline (2) $\bar{\mu}=5.4 \times 10^{+2} ; \bar{\lambda}=4.5 \times 10^{+8}$ & 3 nodes & 14 nodes & 15 nodes \\
\hline (3) $\bar{\mu}=5.4 \times 10^{+2} ; \bar{\lambda}=4.5 \times 10^{+2}$ & 3 nodes & 13 nodes & 16 nodes \\
\hline (4) $\bar{\mu}=5.4 \times 10^{-2} ; \bar{\lambda}=4.5 \times 10^{+8}$ & 0 nodes & 00 nodes & 32 nodes \\
\hline (5) $\bar{\mu}=5.4 \times 10^{-2} ; \bar{\lambda}=4.5 \times 10^{+2}$ & 0 nodes & 00 nodes & 32 nodes \\
\hline (6) $\bar{\mu}=5.4 \times 10^{-2} ; \bar{\lambda}=4.5 \times 10^{-2}$ & 0 nodes & 00 nodes & 32 nodes \\
\hline
\end{tabular}




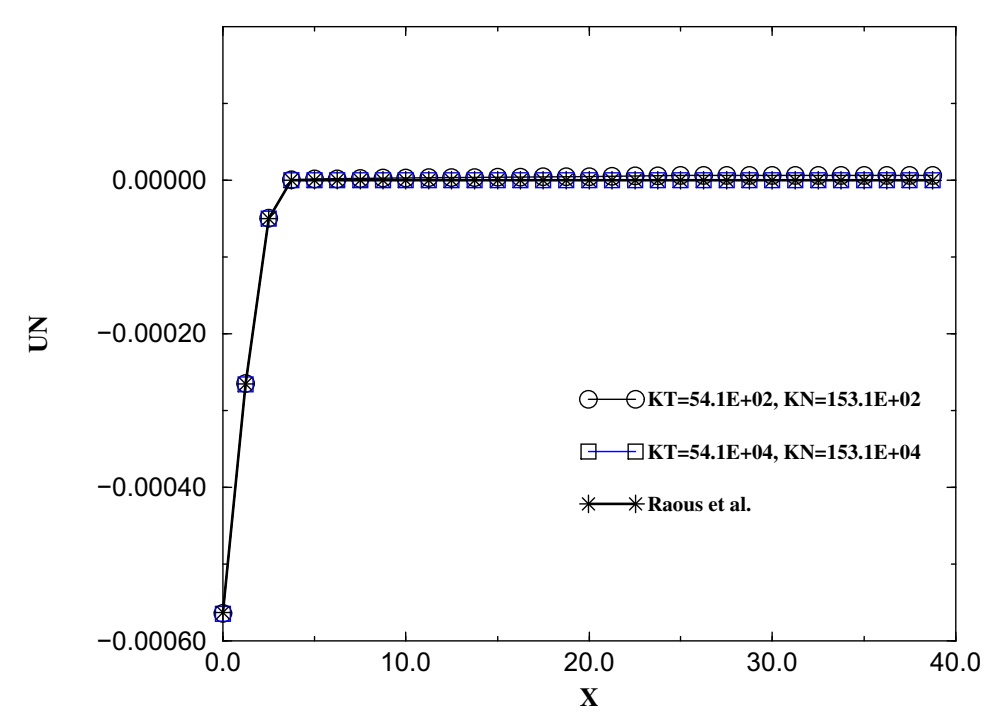

Fig. 13. Normal displacement with respect to $X$.

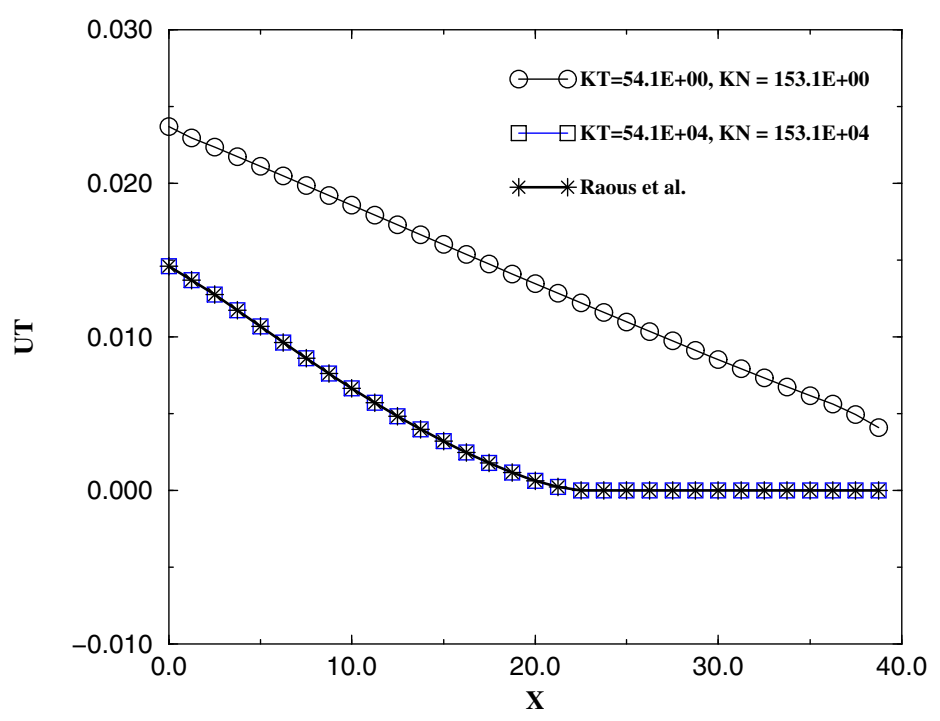

Fig. 14. Tangential displacement with respect to $X$.

The contact zone (interface law) corresponds to the part $A D$ with a friction coefficient equal to $f=1 . u_{1}=0$ on $D E$ and $u_{1}=u_{2}=0$ at point $D$. The loading $F 1=10 \mathrm{daN} / \mathrm{mm}^{2}$ is imposed on $A G$ and $F 2=-5 \mathrm{daN} / \mathrm{mm}^{2}$ on $G E$. Using the finite element method, the contact zone is discretized by 32 nodes. The changes in contact status are given in Table 3 at different values of $\bar{\mu}$ and $\bar{\lambda}$.

If we compare the results obtained with those published in [29] (Signorini-Coulomb laws) it can be seen that at $\bar{\lambda}=4.510^{+8}$ and $\bar{\mu}=5.410^{+8}$ (first line in Table 3), the present results are similar to those obtained by Raous et al. [29]. These coefficients, which are very large (i.e. "equal" to infinity), correspond to the limit case (Signorini-Coulomb). The decrease in the values of $\bar{\mu}$ (lines 1, 2 and 4) corresponds to the increase in the stick zone (13, 15 and 32 nodes). Note that for this example and for cases presented in Fig. 13, that the normal displacements are not changed a lot by the variations of $K_{N}$ and $K_{T}$. In particular, the penetration is negligible. However, as observed on Fig. 14, for this problem the variations of coefficients strongly affect the tangential displacements. A low value of the coefficient $K_{T}$ reflects an increase in the tangential displacement. The nodes slide without reaching the value of Coulomb's sliding limit (Fig. 14). Note that numerical tests have shown the robustness of this algorithms [33]. 


\section{Conclusion}

A method is presented here for modeling a soft thin elastic layer under unilateral contact and dry friction conditions, using asymptotic expansions. Nine contact laws of the compliance kind are obtained and their validity is confirmed. There laws depend on the ratio between the stiffness and thickness parameters of the adhesive. The nine cases are obtained by a comparison with a reference value associated to the rigidity and the geometry of the adherents. These laws were implemented in a Finite Element software program. An academical example was introduced, validated and compared with classical tests [29]. The relaxation algorithm developed for dealing with these laws shows the efficiency and the robustness of this method in these classical tests.

This study should lead to many developments. It would be of interest to extend the results to account for the residual stress and to an adhesive which having a similar rigidity to those of the adherents.

\section{References}

[1] A. Ait-Moussa, Modélisation et étude des singularités d'un joint collé, Ph.D. Thesis, Université Montpellier II, 1989.

[2] O. Allix, P. Ladeveze, Damage analysis of interlaminar fracture specimens, Composite Structures 31 (1995) 61-74.

[3] G. Amontons, De la résistance causée dans les machines, tant par les frottements des parties qui les composent, que par la raideur des cordes qu'on emploie, et la manière de calculer l'un et l'autre, Mémoires de l'Académie Royale des Sciences Paris, 1699.

[4] M. Barquins, D. Wehbi, Study of adherence of elastomers by cyclic unloading experiments, Journal of Adhesion 20 (1986) 55-74.

[5] E. Barthel, On the description of the adhesive contact of spheres with arbitrary interaction potentials, Journal of Colloid and Interface Science 200 (1998) 7-18.

[6] J.-L. Chaboche, R. Girard, P. Levasseur, On the interface debonding models, International Journal of Damage in Mechanics 6 (1997) $220-257$.

[7] C.A. Coulomb, Théorie des machines simples, en ayant égard au frottement de leurs parties, et a la raideur des cordages, Mémoires de mathématique et de physique de l'Académie Royale des Sciences Paris, 1785, pp. 161-342.

[8] G. Duvaut, Equilibre d'un solide élastique avec contact unilatéral et frottement de Coulomb, Compte Rendu Académie des Sciences Série I 290 (1980) $263-265$.

[9] W. Eckhaus, Asymptotic Analysis of Singular Perturbations, North-Holland, 1979.

[10] M. Frémond, Adhesion of solids, Journal of Theoretical and Applied Mechanics 6 (1987) 383-407.

[11] J.M. Georges, Frottement, usure et lubrification, La tribologie ou science des surfaces, in: Sciences et Techniques de l'ingènieur, CNRS, 2000.

[12] G. Geymonat, F. Krasucki, Analyse asymptotique du comportement en flexion de deux plaques collées, Compte Rendu Académie des Sciences Série I 325 (1997) 307-314.

[13] R. Glowinski, J.L. Lions, R. Trémolières, Analyse numérique des inéquations variationnelles, Dunod, 1975.

[14] Z. Hashin, Thin interphase/imperfect interface in elasticity with application to coated fiber composites, Journal of Mechanics and Physics of Solids 50 (2002) 2509-2537.

[15] K.L. Johnson, Contact Mechanics, Cambridge University Press, 1985.

[16] K.L. Johnson, J.A. Greenwood, An adhesion map for the contact of elastic spheres, Journal of Colloid and Interface Science 192 (1997) $326-333$.

[17] K.L. Johnson, K. Kendall, A.D. Roberts, Surface energy and the contact of elastic solids, Proceedings of Royal Society London A 324 (1971) $301-313$.

[18] A. Klarbring, Derivation of the adhesively bonded joints by the asymptotic expansion method, International Journal of Engineering Science 29 (1991) 493-512.

[19] F. Krasucki, A. Mnnch, Y. Ousset, Analyse asymptotique d'un assemblage collé en élasticité non linéaire, Compte Rendu Académie des Sciences Série IIb 329 (2001) 429-434.

[20] F. Lebon, A. Ould-Khaoua, C. Licht, Numerical study of soft adhesively bonded joints in finite elasticity, Computational Mechanics 21 (1997) 134-140.

[21] F. Lebon, M. Raous, Friction modelling of a bolted junction under internal pressure loading, Computers and Structures 43 (1992) $925-933$.

[22] C. Licht, Comportement asymptotique d'une bande dissipative mince de faible rigidité, Compte Rendu Académie des Sciences Série I 317 (1993) $429-$ 433.

[23] C. Licht, G. Michaille, Une modélisation du comportement d’un joint collé élastique, Compte Rendu Académie des Sciences Série I 322 (1996) 295-300.

[24] C. Licht, G. Michaille, A modeling of elastic adhesive bonded joints, Advances in Mathematical Sciences and Applications 7 (1997) $711-740$.

[25] D. Maugis, Contact, Adhesion and Rupture of Elastic Solids, Springer, 2000.

[26] Y. Monerie, M. Raous, Unilateral contact, friction and adhesion: third cracks in composite material, in: J.A.C. Martins, M.D.P. Monteiro Marques (Eds.), Contact Mechanics, 2002.

[27] A.I. Murdoch, Some fundamental aspects of surface modelling, Journal of Elasticity 80 (2005) 33-52.

[28] A. Ould-Khaoua, F. Lebon, C. Licht, G. Michaille, Thin layers in elasticity: a theoretical and numerical study, in: Proceedings of the 1996 ESDA Conference, vol. 4, ASME, 1996, pp. 171-178.

[29] M. Raous, P. Chabrand, F. Lebon, Numerical methods for solving unilateral contact problem with friction, Journal of Theoretical and Applied Mechanics 7 (1988) 111-128.

[30] A.D. Roberts, A.G. Thomas, The adhesion and friction of smooth rubber surfaces, Wear 33 (1975) 45-64.

[31] J. Sanchez-Hubert, E. Sanchez-Palencia, Introduction aux méthodes asymptotiques et à l'homogénisation, Masson, 1992.

[32] P. Suquet, Discontinuities and plasticity, in: Nonsmooth Mechanics and Applications, Springer-Verlag, 1988, pp. 315-323.

[33] F. Zaittouni, Modélisation théorique et numérique d'interfaces, Ph.D. Thesis, Université Montpellier II, 2000.

[34] F. Zaittouni, F. Lebon, C. Licht, Etude théorique et numérique du comportement d'un assemblage de plaques, Compte Rendu Académie des Sciences Série Mécanique 330 (2002) 359-364. 